\title{
A Hybrid Artificial Bee Colony with Differential Evolution
}

\author{
Chukiat Worasucheep
}

\begin{abstract}
Artificial bee colony (ABC) is a relatively new stochastic algorithm with competitive performance and minimal tuning parameter. This paper proposes a hybrid $\mathrm{ABC}$ algorithm with differential evolution (DE), but without additional parameters. DE is a well-known efficient evolutionary algorithm with proven records but its parameter setting is complicated. This proposed hybrid algorithm called $\mathrm{ABCDE}$ incorporates the powerful mutation strategies of $\mathrm{DE}$ into $\mathrm{ABC}$, in order to increase convergence while diversity is not compromised. The performance of $\mathrm{ABCDE}$ is evaluated against both original $\mathrm{ABC}$ and opposition-based DE (ODE), a recent DE variant with high performance. The experiment uses twelve widely accepted non-linear benchmark functions with various characteristics, such as difficult landscape, multimodality, shift and rotation, to evaluate the ABCDE's performance on many complex functions. The experimental results demonstrate a superior performance of $\mathrm{ABCDE}$ against original $\mathrm{ABC}$ and $\mathrm{ODE}$.
\end{abstract}

Index Terms-Artificial bee colony, hybridization, differential evolution.

\section{INTRODUCTION}

Artificial bee colony (ABC) algorithm [1] is a biological-inspired population-based stochastic algorithm, recently proposed by $\mathrm{D}$. Karaboga, which mimics the foraging behavior of honey bee swarm. Performance of ABC algorithm has been demonstrated to be competitive to other population-based algorithms with an advantage of simplicity and having fewer control parameters [2], [3]. Thus ABC has been applied to solve different optimization problems such as machining process [4], scheduling [5], structural design problem [6] and power electric [7]. The ABC employed in this work is a hybrid with differential evolution (DE) in order to increase exploitation, the ability of searching near a candidate solution. DE is a very efficient evolutionary algorithm proposed by Storn and Price [8], whose performance has been improved and widely accepted in many areas from engineering and science to finance and economic since [9]. The hybridization in this work increases ABC's exploitation without additional algorithmic parameters. Performance of the proposed hybrid algorithm, namely ABCDE, is evaluated using a set of widely accepted benchmark of nonlinear minimization problems with different characteristics including multimodality, shift and rotation.

The remainder of the paper has the following structure. Section II presents background of $\mathrm{ABC}$ and overview of DE and ANN. Section III describes the proposed hybrid ABCDE

Manuscript received October 1, 2014; revised December 23, 2014.

C. Worasucheep is with the King Mongkut University of Technology Thonburi, Bangkok, Thailand (e-mail: chukiat.wor@kmutt.ac.th). whose performance is evaluated using benchmark functions in Section IV. Section V concludes the paper with some future works.

\section{BACKGROUND}

Without loss of generality, this paper considers minimization problems only.

\section{A. Artificial Bee Colony}

Artificial Bee Colony (ABC) algorithm is a relatively new population-based algorithm proposed by $\mathrm{D}$. Karaboga in 2005 to solve continuous problems [1]. ABC is modeled from the foraging behavior of honey bee colony that consists of three different groups of bees: employed bees, onlooker bees and scout bees. The employed bees search food sources around the hive and carry information about food source positions. Onlooker bees are waiting in the hive for the information shared by the employed bees about their found food sources, given that each employed bee carries information of one different food source. An onlooker bee evaluates the information showed by the dancing employed bees and choose a food source according to the probability proportional to the quality, the amount of nectar, of that food source. Once an onlooker is attracted to one food source information, it updates food information, keeps only the better one, and shares its information on the dance platform. If a food source could not be improved by the employed or onlooker bees in a certain time (called as abandon limit), the employed bee abandons this food source and becomes a scout bee exploring a new food source.

In ABC algorithm, there are $N$ food sources, each of which is a candidate solution whose position is represented as $x_{i}(i=$ $1, \ldots, N)$ of $D$ dimensional vector, where $D$ is the number of optimization variables. Initially, the population of solutions is created and each $x_{i}$ is randomized with $D$-dimensional real-value vector:

$$
x_{i, j}=x_{\min , j}+\operatorname{rand}(0,1)\left(x_{\max , j}-x_{\min , j}\right)
$$

where $j=1,2, \ldots, D \cdot x_{\min , j}$ and $x_{\max , j}$ are the lower and upper bounds of the dimension $j$, respectively. $\operatorname{rand}(0,1)$ is a random number drawn from a uniform distribution in a range of 0 and 1. Fitness values of all solutions, representing quality of the food sources, are then evaluated. Similar to other population-based algorithms, $\mathrm{ABC}$ is an iterative process and the following steps will be executed repeatedly until some termination criteria is met.

Each employed bee $x_{i}$ generates a new food source $v_{i}$ in the neighborhood of its current position by using the following search equation: 


$$
v_{i, j}=x_{i, j}+\phi_{i, j}\left(x_{i, j}-x_{k, j}\right),
$$

where $k=\{1,2, \ldots, N\}$ and $j=\{1,2, \ldots, D\}$ are randomly chosen indexes; $k$ has to be different from $i . \phi_{i, j}$ is a random number in the range $[-1,1]$. The new food source $v_{i}$ will be evaluated and compared to $x_{i}$. If $v_{i}$ is better than $x_{i}$, the employed bee will memorize the new food source $v_{i}$ and forget the previous one; otherwise $x_{i}$ is retained.

After all employed bees complete their searches, they return to the dancing area in the hive and share information to the onlooker bees. An onlooker chooses a food source depending on the probability value associated with the food source $p_{i}$. In original ABC, roulette wheel selection method is employed as follows.

$$
p_{i}=\text { fit }_{i} / \sum_{j=1}^{N} \text { fit }_{j},
$$

where $f t_{i}$ is the fitness value of solution $i$. The higher value the $\mathrm{fit}_{i}$ is, the more probability that the $i$-th food source is selected. Once an onlooker selects its food source, it produces a modification on the $x_{i}$ by using equation (2) and evaluates the new food source. Similar to the case of the employed bee, an onlooker replaces the selected food source with the new one if the new one is better.

If a food source $x_{i}$ cannot be improved by the bees for a predetermined number of times, called abandon limit, the food source is abandoned and could be newly produced by a scout bee using equation (1).

\section{B. Improved Variants of $A B C$}

It is widely known that both exploration (diversification) and exploitation (intensification) are necessary for any population-based search algorithms. Unfortunately, they are contradict to each other and both must be well balanced during the search for good performance. The original $\mathrm{ABC}$ is well known for its exploration but poor at exploitation[10], [11]. Thus many techniques have been employed to enhance the convergence and performance reliability of $\mathrm{ABC}$. Some of them include local search [5], Tagushi method [6], orthogonal learning strategy [11], multi-strategy ensemble [12]. Kang et al.'s Rosenbrock ABC algorithm [13] used a modified Rosenbrock's rotational direction method to implement the exploitation phase to assist $\mathrm{ABC}$ in solving complex problems Bose et al. [14] proposed the idea of decentralization of attraction from super-fit members along with neighborhood information and wider exploration of search space and applied it to optimal filter design problems.

In additions, hybridization with other well-known population-based algorithms such as particle swarm optimization (PSO) or differential evolution (DE), have been proposed to enhance the performance of $\mathrm{ABC}$ [7], [15]. Inspired by $\mathrm{PSO}$, the gbest-guided $\mathrm{ABC}$ exploits the information of global best solution into the search equation to improve the exploitation [10]. Inspired by DE/best/1, the modified $\mathrm{ABC}$ searches only around the best solution to improve the exploitation [11] in addition to an improved initialization with chaotic system and opposition-based learning. A new probability parameter is added for balancing of the exploration and exploitation. More recently, a hybrid $\mathrm{ABC}$ with $\mathrm{DE}$ integrates a modified $\mathrm{DE}$ into the modified gbest-guided $\mathrm{ABC}$ to further accelerate the convergence [16].
A catastrophe-like scheme is used to prevent stagnation at the later stage of evolution by abruptly initializing some worse individuals of the population. Despite its promising performance, this hybrid $\mathrm{ABC}$ has four additional parameters: two for the improved search equations and two for the stagnation prevention scheme. Yang et al. [17], applied the mutation and crossover strategies of DE to the employed bees to enforce their exploration ability while onlooker bees keep their original updating strategy to retain the exploitation ability. Li and Yin proposed another simple hybrid ABC and $\mathrm{DE}$ where the DE operation works as main structure and $\mathrm{ABC}$ works only when the solution created by DE operator does not make an improvement [18]. Unfortunately, a comprehensive simulation of the algorithms as proposed in Yang et al. [17] and Li and Yin [18] did not achieve a comparable results as reported, therefore, both algorithms are not included in this study for a comparative analysis.

\section{Differential Evolution}

Differential evolution is a population-based stochastic search algorithm that evolves a population of candidate solutions, called vectors, towards global optima. Firstly, a population of vectors are randomly initialized within the search space and evaluated with the objective function provided. Then each vector, so-called target vector, undergoes three operations in sequence: mutation, crossover and replacement [8].

1) Mutation. A base vector is first selected from a randomly chosen or the best vector of the population. Then the difference(s) of one or sometimes two pairs of randomly chosen vectors are scaled and added to the basis vector to produce a mutant vector.

2) Crossover. To enhance the potential diversity of the population, the mutant vector exchanges its components with the target vector to form a trial vector.

3) Selection. The new trial vector and the corresponding target vector will be compared to keep only one of them to survive. If the trial vector has an equal or better value of the fitness value, it replaces the target vector in the next generation; otherwise the target vector is retained. This selection is thus in a greedy way and the overall fitness value will keep better or remain the same [19].

There exist abundant methods developed so far to create the base vector and the difference vectors, which correspond to different mutation strategies. One of the most widely used mutation strategy is DE/rand/1, meaning that one randomly chosen vector is used as the base vector and one pair of randomly chosen vectors are used to create the difference vector in mutation. This strategy provides a moderate to good performance for a wide range of problems. Another popular strategy is DE/best/1 which is similar to DE/rand/1 except that the currently best vector is selected to be the base vector. DE/best/1 strategy yields a good convergence for simple unimodal problems. Many more strategies can be found in a recent survey [9].

An interesting mutation strategy is DE/current-to-rand/1 which is rotationally invariant and has superior performance on difficult problems [9], [19]. DE/current-to-rand/1 replaces the binomial crossover operator with the rotationally invariant arithmetic line recombination operator to generate the trial 
different search behavior. DE/best/1 provides a fast convergence on a wide range of characteristics of functions. $\mathrm{DE} / \mathrm{rand} / 2 /$ dir [20] provides performances of similar quality with $\mathrm{DE} / \mathrm{rand} / 1$ but is slightly faster to converge than $\mathrm{DE} / \mathrm{rand} / 1$ on multimodal and separable functions [9]. Therefore, inspired by DE/best/ 1 and DE/rand/2/dir, the search is around the bee with the best fitness value and the objective function information is incorporated to guide the direction as in the following way:

$$
v_{i, j}=x_{\text {best }, j}+F\left(x_{\text {dest }, j}-x_{s r c, j}\right)
$$

where $x_{b e s t}$ denotes the bee with the currently best fitness value. $x_{s r c}$ and $x_{\text {dest }}$ are the bees chosen with random such that $f\left(x_{d e s t}\right)<f\left(x_{s r c}\right)$, for a minimization problem. $F$ is a random value from a uniform distribution between 0 and 1 .

In addition, after the roulette wheel selection method is performed, the probability for the bee with best fitness ( $\left.p_{\text {best }}\right)$ is set to 1.0 so as to guarantee that the best bee will be updated with the new onlooker equation (6).

All the modifications described here do not introduce additional algorithmic parameters, and hence the strength of the $\mathrm{ABC}$ algorithm is retained.

For the third weakness of equation (2) mentioned earlier, we do not make any improvement in this work. The common idea is to have more dimensions be altered in an iteration, controlled with a newly-introduced probability parameter. By this way the solution is generated more like the crossover operator of DE rather than the $\mathrm{ABC}$ algorithm, and hence we leave for further investigation in next work.

\section{Performance Evaluation}

\section{A. Experimentation Setup}

Twelve benchmark functions are used in the following experiment to evaluate the performance of the proposed hybrid ABCDE algorithm. All these functions are scalable minimization problems widely used in literature and have various characteristics. Table I summarizes their brief characteristics and search ranges. The first six functions are basic unimodal and more difficult multimodal functions. The Sphere function $(f 1)$ is for testing an algorithm's convergence speed on a very simple function. Function 2 is irregular from being altered with the addition of noise. The Rosenbrock function $(f 3)$ has a very narrow valley from local optimum to global optimum. It is considered a multimodal function at a higher dimension than 3 [21]. The Rastrigin function (f6) contains a huge number of local optima dispersed throughout the search space.

Functions 7 to 12 are more difficult forms of selected basic functions by having axis rotation or their optimum shifted. Functions 7 to 9 are selected from the special session and competition held under the IEEE Congress on Evolutionary Computation (CEC) 2005 while functions 9 to 12 are selected from CEC 2013. Details of the benchmark set are given in the corresponding technical reports [22], [23].

The experiment is conducted on all these 12 functions at 30 dimensions (or the number of decision variables). The maximum number of function evaluations (MAXNFC) is set to 200000. All experiments were run 60 times independently. Mean, s.d., highest value and lowest values of the final fitness obtained from 60 runs by the proposed $\mathrm{ABCDE}$ are compared with those by original $\mathrm{ABC}$ as well as opposition-based $\mathrm{DE}$ (ODE) [24]. ODE is a state-of-the-art DE enhanced with the opposition-based learning in population initialization, generation jumping, and local improvement. Despite its simple implementation, performance of ODE was comprehensively proven using a large set of complex benchmark functions [24].

Population size (PS) is known to have some impact to the search diversity and on the performance of population-based algorithms. PS for ABCDE and $\mathrm{ABC}$ are set equally to 20 and 60 . PS for a DE is recommended widely from $3 \cdot D$ to $10 \cdot D[8]$, [9]. In this experiment, we tested ODE at 60, 90, 120, 150 and 180 to investigate the results and found that the overall best results are obtained from PS $=60$ and 90, and thus will only be reported in the next section. Other important parameters for ODE are as follows: $C R=0.85, F=0.5$, and jumping rate $J_{R}=0.3$, as recommended [24]. The abandon limit for both $\mathrm{ABC}$ and ABCDE is set to 0.6 $\mathrm{PS} \cdot D$ as recommended [11].

All algorithms are implemented in Java 7 using NetBeans IDE 7.3 and executed on a computer running Windows 7 SP1 with an Intel i7 Quad Core 3.40 GHz.

\section{B. Comparison Results and Discussions}

Table II lists the means, standard deviations (s.d.), the lowest and the highest fitness obtained from running each algorithm for 60 times. Any values smaller than 1e-80 are reported as 0 . The lowest mean and s.d. values indicate best performance of algorithm for that function and are highlighted in bold. Table III summarizes statistical ranking of final fitness values obtained for each benchmark function; a lower-number rank is better. To rank algorithms for each function, the two-tailed Wilcoxon signed-rank test, a well-known nonparametric statistical hypothesis test, is conducted to test the difference in final fitness values obtained from a pair of algorithms at 0.05 significant level. An algorithm $j$ is ranked better than algorithm $k\left(r_{j}<r_{k}\right)$ if the Wilcoxon signed-rank test result of algorithms $j$ against $k$ gets a $p$-value below 0.05 . Two algorithms are ranked equally if the Wilcoxon signed-rank test result is not significant. Then the ranks of each algorithm are averaged by function groups: a group of six basic functions and a group of six complex functions (with shift and rotation) as well as all twelve functions, to obtain the Average Aggregated Ranks (AAR). A lower value of AAR means that such algorithm performs better. The last column (\#1) in Table III indicates the number of functions, in which each algorithm gets the first rank. A higher value of \#1 means the algorithm is better.

Fig. 1 and Fig. 2 display the average convergence graphs of all algorithms-PS's for each function. To avoid overcrowding in the graphs, the convergence graphs of ODE with PS $=60$ (ODE-60) are omitted since it has a lower performance in overall compared to ODE-90.

Last column in Table II shows the running time (in seconds) for 60 runs of each algorithm as a comparison. It is clear that 
ODE took a longer average time than $\mathrm{ABC}$ and $\mathrm{ABCDE}$ to finish its running (with MAXNFC) in every function. This is due to the overhead time for sorting in the ODE's opposition-based learning. In addition, we notice that on average $\mathrm{ABCDE}$ and $\mathrm{ABC}$ took about the same time to run.

\section{1) Basic functions}

From Table II and Fig. 1 (a) and (b), both ABCDE and ODE clearly outperforms original $\mathrm{ABC}$ on basic unimodal functions $(f 1$ and $f 2)$. ABCDE with $\mathrm{PS}=20$ (ABCDE-20) has the fastest convergence in $f 1$, but ODE wins in the case of noisy function $(f 2)$.

Functions $f 3$ to $f 6$ are more difficult multimodal functions. ABCDE clearly outperforms ODE and beats original ABC in $f 3$ Rosenbrock and f6 Rastrigin functions which are very challenging. Every algorithm can achieve a minimum value of 0 for Griewank function. Although on average ODE-90 is better than all $\mathrm{ABC}$ variants in Ackley and Griewank functions, ODE-60 performs the worst. ABCDE performs better than $\mathrm{ABC}$ in both functions.

Considering the AAR columns in Table III, we can observe that $\mathrm{ABCDE}$ achieves the top two best $\mathrm{AAR}_{B A S}$, i.e. 2.33 and
2.67 for ABCDE-20 and ABCDE-60, respectively.

From Fig. 1, we can see that ABCDE-20 converges fastest for 5 out of 6 functions. The improved solution generations of both employed bees and onlooker bees significantly accelerate their convergence, while the main structure of bee colony algorithm and its abandon limit help preserve the exploration capability.

\section{2) Complex functions}

For shifted functions $(f 7-f 9)$ according to Table III, both $\mathrm{ABC}$ and $\mathrm{ABCDE}$ perform equally in terms of rank regardless of population size and clearly beat ODE. For rotated functions $(f 10-f 12)$, the winners are $\mathrm{ABC}-60$ and $\mathrm{ABCDE}-20$ with equal $\mathrm{AAR}_{C P X}$ of 1.17 .

From Fig. 2, the convergence of ODE is faster than other algorithms only in $f 11$ Rotated Griewank function. However, ABCDE-20 converges the fastest for 3 out of 6 complex functions.

In summary for all twelve functions, ABCDE-20 achieved the best (lowest) AAR (=1.75) as well as the highest number of \#1 (= 7). This confirms the competitiveness of the proposed hybrid ABCDE.

TABLE II: BASIC STATISTICS OF THE RESUlTS AND RUNNING TIME (IN SECONDS) ANY VALUES SMALLER THAN 1E-80 ARE REPORTED AS 0

\begin{tabular}{|c|c|c|c|c|c|c|c|}
\hline$\#$ & Algo. & PS & Mean & s.d. & Lowest & Highest & Time \\
\hline \multirow[t]{6}{*}{$f 1$} & $\mathrm{ABC}$ & 20 & 5.924E-78 & $3.182 \mathrm{E}-77$ & 0 & $2.456 \mathrm{E}-76$ & 1.04 \\
\hline & & 60 & $2.883 \mathrm{E}-23$ & $8.286 \mathrm{E}-23$ & $1.212 \mathrm{E}-25$ & $6.250 \mathrm{E}-22$ & 1.08 \\
\hline & ABCDE & 20 & 0 & 0 & 0 & 0 & 1.03 \\
\hline & & 60 & $1.165 \mathrm{E}-35$ & 4.207E-35 & $2.131 \mathrm{E}-39$ & $2.486 \mathrm{E}-34$ & 1.05 \\
\hline & ODE & 60 & $1.665 \mathrm{E}-04$ & $1.274 \mathrm{E}-03$ & 0 & $9.954 \mathrm{E}-03$ & 5.02 \\
\hline & & 90 & 0 & 0 & 0 & 0 & 5.40 \\
\hline \multirow[t]{6}{*}{$f 2$} & $\mathrm{ABC}$ & 20 & $5.486 \mathrm{E}-02$ & $1.069 \mathrm{E}-02$ & $2.977 \mathrm{E}-02$ & 7.998E-02 & 6.88 \\
\hline & & 60 & $6.526 \mathrm{E}-02$ & 1.449E-02 & $4.075 \mathrm{E}-02$ & $1.123 \mathrm{E}-01$ & 6.96 \\
\hline & ABCDE & 20 & 2.903E-02 & $6.534 \mathrm{E}-03$ & $1.201 \mathrm{E}-02$ & 4.300E-02 & 7.01 \\
\hline & & 60 & $2.431 \mathrm{E}-02$ & $4.746 \mathrm{E}-03$ & $1.255 \mathrm{E}-02$ & $3.737 \mathrm{E}-02$ & 7.03 \\
\hline & ODE & 60 & $6.588 \mathrm{E}-04$ & 2.848E-04 & $3.475 \mathrm{E}-04$ & 2.319E-03 & 14.02 \\
\hline & & 90 & $9.450 \mathrm{E}-04$ & $2.841 \mathrm{E}-04$ & $3.765 \mathrm{E}-04$ & $1.598 \mathrm{E}-03$ & 14.11 \\
\hline \multirow[t]{6}{*}{$f 3$} & $\mathrm{ABC}$ & 20 & 0.260 & 0.303 & $2.72 \mathrm{E}-02$ & 1.877 & 12.35 \\
\hline & & 60 & 0.438 & 1.192 & 4.74E-03 & 9.005 & 12.47 \\
\hline & ABCDE & 20 & 0.494 & 1.472 & $7.58 \mathrm{E}-05$ & 10.028 & 12.53 \\
\hline & & 60 & 0.183 & 0.298 & $5.67 \mathrm{E}-03$ & 1.597 & 12.59 \\
\hline & ODE & 60 & 27.143 & 0.529 & $2.54 \mathrm{E}+01$ & 27.969 & 23.02 \\
\hline & & 90 & 26.805 & 0.416 & 25.595 & 27.689 & 23.12 \\
\hline \multirow[t]{6}{*}{$f 4$} & $\mathrm{ABC}$ & 20 & $4.266 \mathrm{E}-11$ & $2.915 \mathrm{E}-11$ & $1.030 \mathrm{E}-11$ & $1.299 \mathrm{E}-10$ & 4.02 \\
\hline & & 60 & $3.946 \mathrm{E}-14$ & $4.228 \mathrm{E}-15$ & $2.887 \mathrm{E}-14$ & $5.018 \mathrm{E}-14$ & 4.10 \\
\hline & ABCDE & 20 & $3.147 \mathrm{E}-14$ & $2.817 \mathrm{E}-15$ & $2.887 \mathrm{E}-14$ & $3.952 \mathrm{E}-14$ & 3.99 \\
\hline & & 60 & $3.183 \mathrm{E}-14$ & $3.122 \mathrm{E}-15$ & $2.176 \mathrm{E}-14$ & $3.952 \mathrm{E}-14$ & 4.03 \\
\hline & ODE & 60 & $2.078 \mathrm{E}-06$ & $1.239 \mathrm{E}-05$ & $3.997 \mathrm{E}-15$ & $9.386 \mathrm{E}-05$ & 10.02 \\
\hline & & 90 & $3.997 \mathrm{E}-15$ & 3.155E-30 & $3.997 \mathrm{E}-15$ & $3.997 \mathrm{E}-15$ & 10.08 \\
\hline \multirow[t]{6}{*}{$f 5$} & $\mathrm{ABC}$ & 20 & $5.351 \mathrm{E}-11$ & $3.366 \mathrm{E}-10$ & 0 & $2.620 \mathrm{E}-09$ & 3.92 \\
\hline & & 60 & $6.077 \mathrm{E}-09$ & $4.029 \mathrm{E}-08$ & 0 & $3.130 \mathrm{E}-07$ & 4.02 \\
\hline & ABCDE & 20 & 4.003E-09 & $1.080 \mathrm{E}-08$ & 0 & $5.907 \mathrm{E}-08$ & 4.02 \\
\hline & & 60 & $3.574 \mathrm{E}-08$ & $1.994 \mathrm{E}-07$ & 0 & $1.552 \mathrm{E}-06$ & 4.05 \\
\hline & ODE & 60 & $8.008 \mathrm{E}-04$ & $2.790 \mathrm{E}-03$ & 0 & $1.232 \mathrm{E}-02$ & 10.02 \\
\hline & & 90 & 0 & 0 & 0 & 0 & 10.05 \\
\hline \multirow[t]{6}{*}{ f6 } & $\mathrm{ABC}$ & 20 & $2.43 \mathrm{E}-13$ & 8.29E-13 & 0 & $6.01 \mathrm{E}-12$ & 3.52 \\
\hline & & 60 & 0 & 0 & 0 & 0 & 3.59 \\
\hline & ABCDE & 20 & 0 & 0 & 0 & 0 & 4.04 \\
\hline & & 60 & 0 & $\mathbf{0}$ & 0 & 0 & 4.13 \\
\hline & ODE & 60 & 61.137 & 43.580 & 2.985 & 140.020 & 10.50 \\
\hline & & 90 & 79.979 & 42.117 & 6.965 & 135.869 & 10.52 \\
\hline$f 7$ & $\mathrm{ABC}$ & 20 & -450 & 0 & -450 & -450 & 0.53 \\
\hline
\end{tabular}




\begin{tabular}{|c|c|c|c|c|c|c|c|}
\hline$\#$ & Algo. & PS & Mean & s.d. & Lowest & Highest & Time \\
\hline & \multirow{3}{*}{$\mathrm{ABCDE}$} & 60 & -450 & O & -450 & -450 & 0.58 \\
\hline & & 20 & -450 & 0 & -450 & -450 & 1.02 \\
\hline & & 60 & -450 & $\mathbf{0}$ & -450 & -450 & 1.08 \\
\hline & \multirow[t]{2}{*}{ ODE } & 60 & -449.992 & 0.0403 & -450 & -449.715 & 5.52 \\
\hline & & 90 & -450 & $\mathbf{0}$ & -450 & -450 & 5.55 \\
\hline \multirow[t]{6}{*}{$f 8$} & \multirow[t]{2}{*}{$\mathrm{ABC}$} & 20 & 395.234 & 6.088 & 390.668 & 428.030 & 0.52 \\
\hline & & 60 & 399.310 & 13.346 & 390.037 & 448.613 & 0.55 \\
\hline & \multirow[t]{2}{*}{$\mathrm{ABCDE}$} & 20 & 400.095 & 14.787 & 390.005 & 448.380 & 1.03 \\
\hline & & 60 & 393.631 & 6.723 & 390.049 & 427.358 & 1.09 \\
\hline & \multirow[t]{2}{*}{ ODE } & 60 & $2.22 \mathrm{E}+06$ & $1.51 \mathrm{E}+07$ & 416.494 & $1.18 \mathrm{E}+08$ & 5.11 \\
\hline & & 90 & $2.90 \mathrm{E}+04$ & $2.13 \mathrm{E}+05$ & 414.890 & $1.67 \mathrm{E}+06$ & 5.50 \\
\hline \multirow[t]{6}{*}{$f 9$} & \multirow[t]{2}{*}{$\mathrm{ABC}$} & 20 & -330 & 0 & -330 & -330 & 4.00 \\
\hline & & 60 & -330 & 0 & -330 & -330 & 4.07 \\
\hline & \multirow[t]{2}{*}{$\mathrm{ABCDE}$} & 20 & -330 & $\mathbf{0}$ & -330 & -330 & 4.01 \\
\hline & & 60 & -330 & 0 & -330 & -330 & 4.09 \\
\hline & \multirow[t]{2}{*}{ ODE } & 60 & -254.038 & 43.933 & -317.833 & -187.514 & 10.01 \\
\hline & & 90 & -246.656 & 42.790 & -316.071 & -179.829 & 10.50 \\
\hline \multirow[t]{6}{*}{$f 10$} & \multirow[t]{2}{*}{$\mathrm{ABC}$} & 20 & -679.029 & 0.053 & -679.186 & -678.932 & 55.93 \\
\hline & & 60 & -679.047 & 0.054 & -679.204 & -678.954 & 57.60 \\
\hline & \multirow[t]{2}{*}{$\mathrm{ABCDE}$} & 20 & -679.042 & 0.045 & -679.215 & -678.972 & 56.01 \\
\hline & & 60 & -679.041 & 0.051 & -679.168 & -678.953 & 57.69 \\
\hline & \multirow[t]{2}{*}{ ODE } & 60 & -679.044 & 0.056 & -679.241 & -678.941 & 76.94 \\
\hline & & 90 & -679.032 & 0.051 & -679.181 & -678.939 & 78.97 \\
\hline \multirow[t]{6}{*}{$f 11$} & \multirow[t]{2}{*}{$\mathrm{ABC}$} & 20 & -493.331 & 1.757 & -497.818 & -488.195 & 15.13 \\
\hline & & 60 & -498.937 & 0.248 & -499.515 & -498.415 & 15.47 \\
\hline & \multirow[t]{2}{*}{$\mathrm{ABCDE}$} & 20 & -499.535 & 0.281 & -499.922 & -498.764 & 15.44 \\
\hline & & 60 & -497.398 & 0.686 & -498.554 & -495.624 & 15.59 \\
\hline & \multirow[t]{2}{*}{ ODE } & 60 & -499.290 & 0.664 & -499.971 & -497.192 & 23.70 \\
\hline & & 90 & -499.944 & 0.075 & -500.000 & -499.483 & 24.15 \\
\hline \multirow[t]{6}{*}{$f 12$} & \multirow[t]{2}{*}{$\mathrm{ABC}$} & 20 & 18011.0 & 2628.9 & 13160.6 & 22328.5 & 7.31 \\
\hline & & 60 & 20245.9 & 2985.1 & 13113.6 & 25769.6 & 7.81 \\
\hline & \multirow[t]{2}{*}{$\mathrm{ABCDE}$} & 20 & 20352.9 & 3470.1 & 13079.9 & 27297.3 & 7.33 \\
\hline & & 60 & 23668.0 & 2780.6 & 18127.2 & 30102.9 & 7.95 \\
\hline & \multirow[t]{2}{*}{ ODE } & 60 & 34353.0 & 24.6 & 34343.9 & 34485.2 & 14.29 \\
\hline & & 90 & 34348.4 & 2.5 & 34344.9 & 34358.3 & 14.31 \\
\hline
\end{tabular}

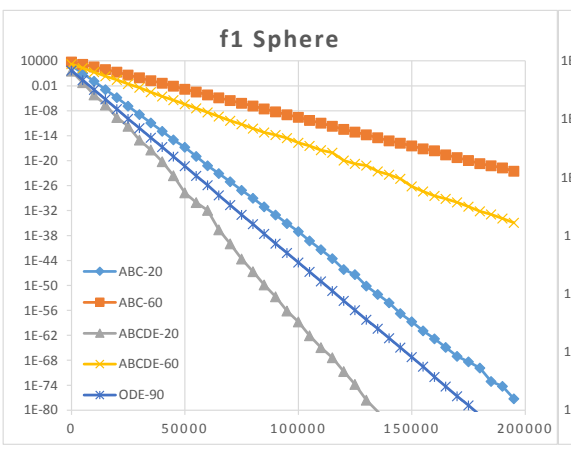

(a)

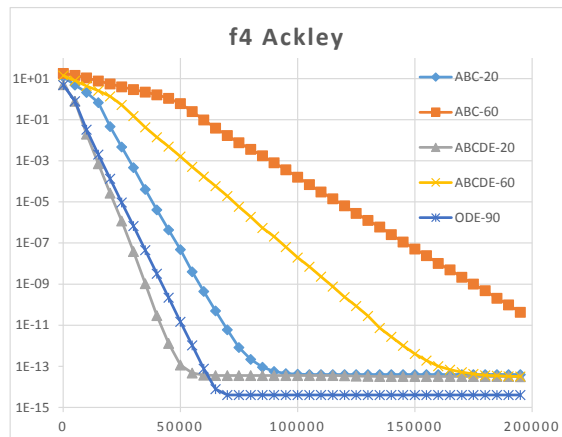

(d)

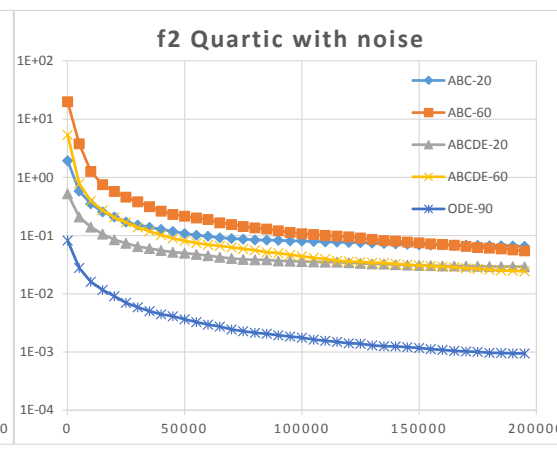

(b)

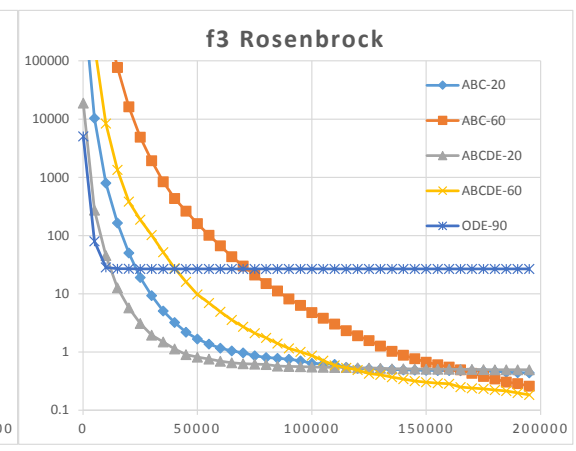

(c)

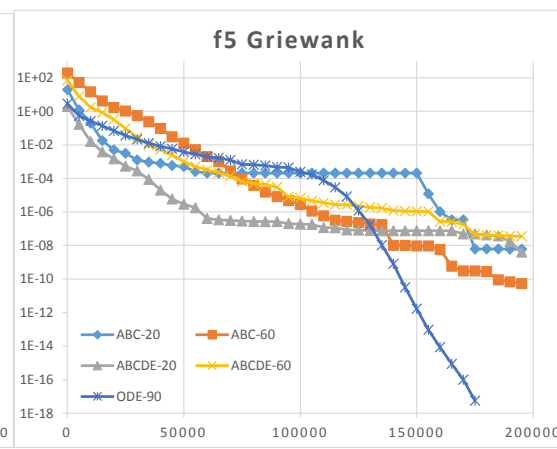

(e)

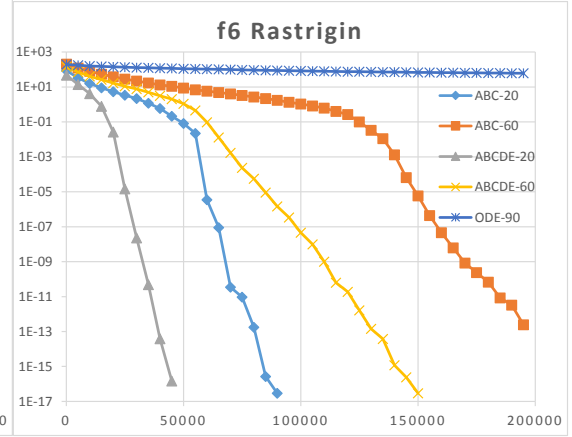

(f)

Fig. 1. Average convergence performance of $\mathrm{ABC}, \mathrm{ABCDE}$ and $\mathrm{ODE}$ from running of 60 times for minimization of basic functions. The number after dash (-) indicates population size. 
TABLE III: RANKS OF THE ALGORITHMS FOR EACH FUNCTION

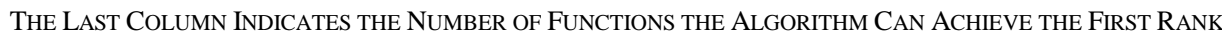

\begin{tabular}{|c|c|c|c|c|c|c|c|c|c|c|c|c|c|c|c|c|c|}
\hline \multirow[t]{2}{*}{ Algorithm } & \multirow[t]{2}{*}{ PS } & \multicolumn{7}{|c|}{ Basic } & \multicolumn{7}{|c|}{ Complex } & \multirow[t]{2}{*}{$A A R$} & \multirow[t]{2}{*}{$\# 1$} \\
\hline & & $f 1$ & $f 2$ & $f 3$ & $f 4$ & $f 5$ & $f 6$ & $A A R_{\mathrm{BAS}}$ & f7 & $f 8$ & $f 9$ & $f 10$ & $f 11$ & $f 12$ & $A A R_{\mathrm{CPX}}$ & & \\
\hline \multirow[t]{2}{*}{$\mathrm{ABC}$} & 20 & 3 & 5 & 2 & 5 & 2 & 4 & 3.50 & 1 & 1 & 1 & 1 & 6 & 1 & 1.83 & 2.67 & 5 \\
\hline & 60 & 5 & 5 & 4 & 2 & 3 & 1 & 3.33 & 1 & 1 & 1 & 1 & 1 & 2 & 1.17 & 2.25 & 6 \\
\hline \multirow[t]{2}{*}{$\mathrm{ABCDE}$} & 20 & 1 & 3 & 4 & 2 & 3 & 1 & 2.33 & 1 & 1 & 1 & 1 & 1 & 2 & 1.17 & 1.75 & 7 \\
\hline & 60 & 4 & 3 & 1 & 2 & 5 & 1 & 2.67 & 1 & 1 & 1 & 1 & 5 & 4 & 2.17 & 2.42 & 6 \\
\hline \multirow[t]{2}{*}{ ODE } & 60 & 6 & 1 & 6 & 6 & 6 & 5 & 5.00 & 6 & 5 & 5 & 1 & 1 & 5 & 3.83 & 4.42 & 3 \\
\hline & 90 & 1 & 2 & 6 & 4 & 1 & 5 & 3.17 & 1 & 5 & 6 & 1 & 1 & 5 & 3.17 & 3.17 & 5 \\
\hline
\end{tabular}

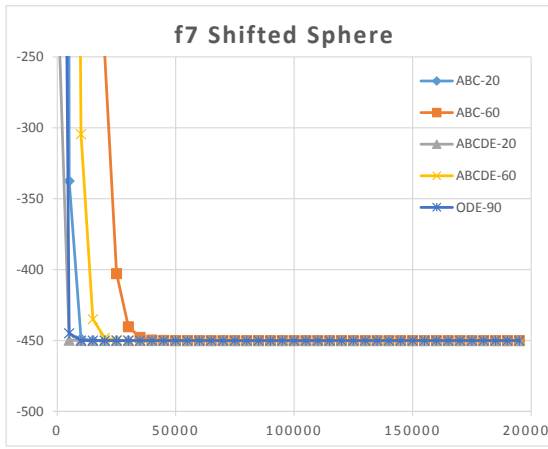

(a)

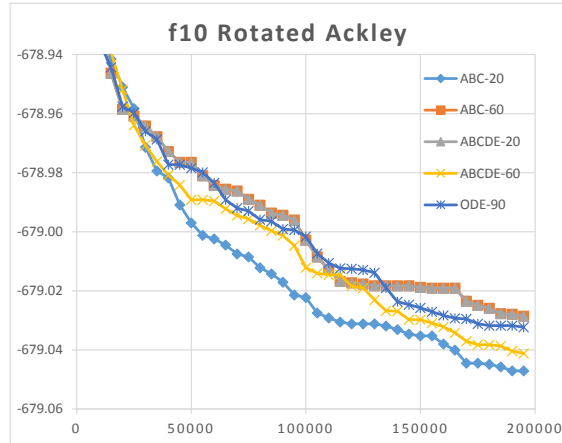

(d)

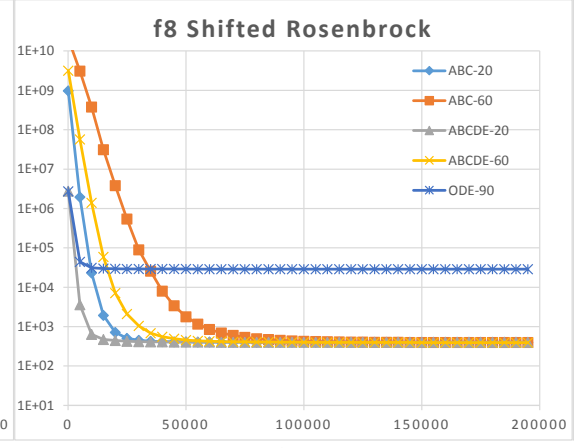

(b)

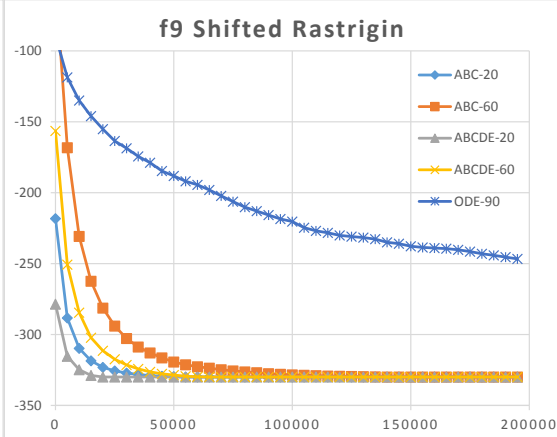

(c)

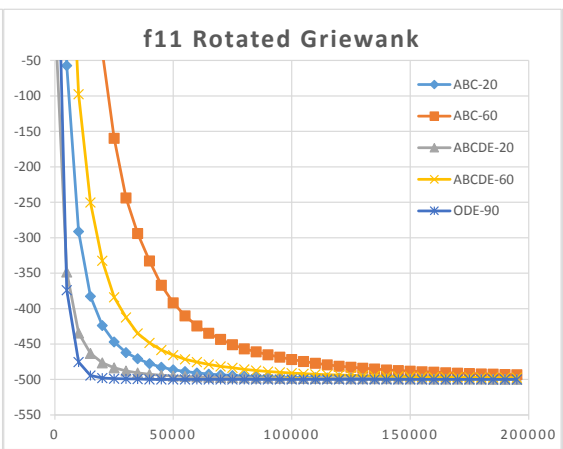

(e)

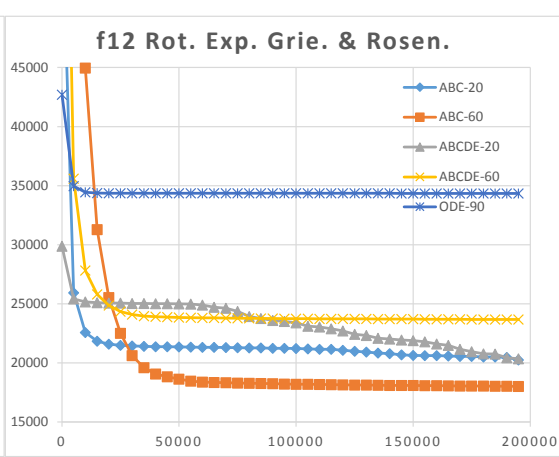

(f)

Fig. 2. Average convergence performance of $\mathrm{ABC}, \mathrm{ABCDE}$ and $\mathrm{ODE}$ from running of 60 times for minimization of complex functions. The number after dash (-) indicates population size.

\section{CONCLUSION}

This paper proposes a hybrid Artificial Bee Colony algorithm with Differential Evolution, called ABCDE. The proposed ABCDE takes advantage of some advance DE's mutation strategies to speed up search convergence while the exploration capability is still maintained and no additional parameters are introduced. An experiment is conducted to evaluate the performance of $\mathrm{ABCDE}$ against original $\mathrm{ABC}$ and ODE, a highly competent DE, with twelve widely accepted benchmark of non-linear minimization problems. The experimental results indicate that the proposed ABCDE has a significant improved convergence speed not only on simple unimodal and multimodal functions but also on rotated and shifted functions. Future works include a more systematic hybridization of $\mathrm{ABC}$ with DE, PSO and other meta-heuristic algorithms with an application of optimizing neural networks for financial applications.

\section{REFERENCES}

[1] D. Karaboga, "An idea based on honey bee swarm for numerical optimization," Erciyes University, Kayseri, Turkey, Technical Report-TR06, 2005.
[2] D. Karaboga and B. Basturk, "On the performance of Artificial Bee Colony (ABC) algorithm," Applied Soft Computing, vol. 8, pp. 687-697, 2008

[3] D. Karaboga and B. Akay, "A comparative study of Artificial Bee Colony algorithm," Applied Mathematics and Computation, vol. 214, pp. 108-132, 2009.

[4] S. Samanta and S. Chakraborty, "Parametric optimization of some non-traditional machining processes using Artificial Bee Colony algorithm," Engineering Applications of Artificial Intelligence, vol. 24, pp. 946-957, 2011.

[5] S. Sundar and A. Singh, "A swarm intelligence approach to early/tardy scheduling problem," Swarm and Evolutionary Computation, vol. 4, no. 1, pp. 25-32, 2012.

[6] A. R. Yildiz, "A new hybrid Artificial Bee Colony algorithm for robust optimal design and manufacturing," Applied Soft Computing, vol. 13, no. 5, pp. 2906-2912, 2013.

[7] P. Lu, J. Zhou, H. Zhang, R. Zhang, and C. Wang, "Chaotic differential bee colony optimization algorithm for dynamic economic dispatch problem with valve-point effects," Electrical Power and Energy Systems, vol. 62, pp. 130-143, 2014.

[8] R. Storn and K. Price, "Differential evolution - A simple and efficient heuristic for global optimization over continuous spaces," Journal of Global Optimization, vol. 11, no. 4, pp. 341-359, 1997.

[9] S. Das and P. N. Suganthan, "Differential evolution - A survey of the state-of-the-art," IEEE Trans. Evolutionary Computation, vol. 15, no. 1, pp. 4-31, Feb. 2011.

[10] G. Zhu and S. Kwong, "Gbest-guided Artificial Bee Colony algorithm for numerical function optimization," Applied Mathematics and Computation, vol. 217, no. 7, pp. 3166-3173, Dec. 2010. 
[11] W. Gao and S. Liu, "A modified Artificial Bee Colony algorithm," Computers and Operation Research, vol. 39, no. 3, pp. 687-697, Mar. 2012.

[12] H. Wang, Z. Wu, S. Rahnamayan, H. Sun, Y. Liu, and J.-S. Pan, "Multi-strategy ensemble Artificial Bee Colony algorithm," Information Sciences, vol. 279, pp. 587-603, 2014.

[13] F. Kang, J. J. Li, and Z. Y. Ma, "Rosenbrock Artificial Bee Colony algorithm for accurate global optimization of numerical functions," Information Sciences, vol. 12, pp. 3508-3531, 2011.

[14] D. Bose, S. Biswas, A. V. Vasilakos, and S. Laha, "Optimal filter design using an improved artificial bee colony algorithm," Information Sciences, vol. 281, pp. 443-461, 2014.

[15] M. S. Kıran and M. Gündüz, "A recombination-based hybridization of particle swarm optimization and artificial bee colony algorithm for continuous optimization problems," Applied Soft Computing, vol. 13, no. 4, pp. 2188-2203, Apr. 2013.

[16] W. Xiang, S. Ma, and M. An, "hABCDE: A hybrid evolutionary algorithm based on Artificial Bee Colony algorithm and differential evolution," Applied Mathematics and Computation, vol. 238, pp. 370-386, 2014.

[17] J. Yang, W. T. Li, X.-W. Shi, L. Xin, and J. F. Yu, "A hybrid ABC-DE algorithm and its application for time-modulated arrays pattern synthesis," IEEE Transactions on Antennas and Propagation, vol. 61 , no. 11, pp. 5485-5495, Nov. 2013.

[18] X. Li and M. Yin, "Hybrid differential evolution with artificial bee colony and its application for design of a reconfigurable antenna array with discrete phase shifters," Microwaves, Antennas \& Propagation, IET, vol. 6, no. 14, pp. 1573-1582, 2012.

[19] K. V. Price, "An introduction to Differential Evolution," in New Ideas in Optimization, D. Corne, M. Dorigo, and F. Glover, Eds. London, U.K.: McGraw-Hill, 1999, pp. 79-108.
[20] V. Feoktistov and S. Janaqi, "Generalization of the strategies in Differential Evolution," in Proc. 18th Parallel and Distributed Processing Symposium, Apr. 2004, p. 165.

[21] Y. W. Shang and Y. H. Qiu, "A note on the extended Rosenbrock function," Evolutionary Computation, vol. 14, no. 1, pp. 119-126, March 2006

[22] P. N. Suganthan, N. Hansen, J. J. Liang, K. Deb, Y. P. Chen, A. Auger, and S. Tiwari, "Problem definitions and evaluation criteria for the CEC 2005 special session on real-parameter optimization," Nanyang Technol. Univ., Singapore, Tech. Rep., IIT Kanpur, Kanpur, India, KanGAL Rep. \#2005005, May 2005.

[23] J. J. Liang, B. Y. Qu, P. N. Suganthan, and A. G. Hernandez-Diaz, "Problem definitions and evaluation criteria for the CEC 2013 special session on real-parameter optimization," Com. Intel. Lab., Zhengzhou University, Zhengzhou, China, Tech. Rep. 201212 and Nanyang Technological University, Singapore, Tech. Rep., 2013.

[24] S. Rahnamayan, H. R. Tizhoosh, and M. M. A. Salama, "Opposition-based differential evolution," IEEE Transactions on Evolutionary Computation, vol. 12, no. 1, pp. 64-79, Jan. 2008.

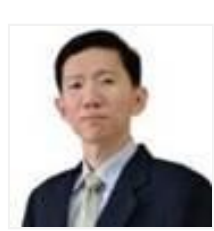

Chukiat Worasucheep obtained a degree of M.S. in computer science from Oregon State University, USA and has been working with Applied Computer Science, Faculty of Science, King Mongkut University of Technology Thonburi, Bangkok, Thailand. His current research interests are computational intelligence, particularly in evolutionary computation and swarm intelligence for financial applications and engineering applications. His recent book is Modern Operating systems (in Thai) to be published in early 2015 . 